\title{
BEYOND DELAUNAY SURFACES
}

\author{
PETER A. DJONDJOROV, MARIANA TS. HADZHILAZOVA \\ IVAÏLO M. MLADENOV AND VASSIL M. VASSILEV
}

Presented by Ivaïlo M. Mladenov

\begin{abstract}
An interesting class of axially symmetric surfaces, which generalizes Delaunay's unduloids and provides solutions of the shape equation is described in explicit parametric form. This class provide the first analytical examples of surfaces with periodic curvatures studied by K. Kenmotsu and leads to some unexpected relationships among Jacobian elliptic functions and their integrals.
\end{abstract}

\section{Introduction}

It is well-known that in aqueous solution, amphiphilic molecules (e.g., phospholipids) form spontaneously bilayers so that the hydrophilic heads of these molecules are located in both outer sides of the bilayer which are in contact with the liquid, while their hydrophobic tails remain at the interior. In many cases, the bilayer form a closed membrane, which is called a vesicle. Vesicles constitute well-defined and sufficiently simple model systems for studying basic physical properties of the more complex biological cells.

In 1973, Helfrich [3] had proposed the so-called spontaneous curvature model according to which the equilibrium shapes of a lipid vesicle are determined by the extremals of the curvature (shape) energy

$$
\mathcal{F}_{c}=\frac{k_{c}}{2} \int_{\mathcal{S}}(2 H-\mathbb{h})^{2} \mathrm{~d} A+k_{G} \int_{\mathcal{S}} K \mathrm{~d} A
$$

under the constraints of fixed total enclosed volume $V$ and area $A$ of its middle surface $\mathcal{S}$. In the above equation $H$ and $K$ denote the mean, respectively the Gaussian curvature of the surface $\mathcal{S}$ while $\mathrm{h}, k_{c}$ and $k_{G}$ are real constants representing the spontaneous curvature, bending and Gaussian rigidity of the membrane. Using two Lagrange multipliers $\lambda$ and $p$, this yields the functional

$$
\mathcal{F}=\mathcal{F}_{c}+\lambda \int_{\mathcal{S}} \mathrm{d} A+p \int \mathrm{d} V
$$


The Lagrangian multipliers $\lambda$ and $p$ are interpreted as tensile stress and pressure difference between the outer and inner media.

The Euler-Lagrange equation corresponding to the functional $\mathcal{F}$ reads

$$
\Delta H+(2 H-\mathbb{h})\left(H^{2}+\frac{\mathfrak{h}}{2} H-K\right)-\frac{\lambda}{k_{c}} H+\frac{p}{2 k_{c}}=0
$$

where $\Delta$ is the Laplace-Beltrami operator on the surface $\mathcal{S}$. Equation (1), that has been derived by Ou-Yang and Helfrich [19], is often referred to as the general membrane shape equation. Its derivation from geometrical standpoint can be found in [26].

In parallel two other curvature models have been developed. The first of them is the so-called bilayer-couple model suggested by Svetina and Žekš in [25] on the ground of the bilayer-couple hypothesis [23] and the related work [24]. The second one is known as the area-difference-elasticity model $[1,12,31]$. For the purposes of the present paper, however, it is important to underline that all the curvature models mentioned above lead to the same set of stationary shapes, determined locally by the equation (1) given above, since they differ only by global energy terms (see $[10,12,23])$. Of course, the meaning of the constants involved in this equation vary within different models. For more than three decades, the study of the equilibrium shapes of the vesicles has attracted much attention, nevertheless only a few analytic solutions to the shape equation (1) have been reported up to now. These are solutions determining: spheres and circular cylinders [19], Clifford tori [5, 20,21], Delaunay surfaces [13, 16], circular biconcave discoids [15, 17], nodoidlike and unduloidlike shapes [16], some types of Willmore and constant squared mean curvature surfaces $[9,29,32]$ as well as cylindrical surfaces [22,27]. It should be noted, however, that, leaving aside the first two types of the aforementioned surfaces whose parametric equations are well known, explicit parametrizations of the rest ones are missing except for the surfaces of Delaunay $[13,14]$ and the generalized cylindrical surfaces [27]. Strangely enough, the rotational ellipsoids furnish only approximate solutions to the shape equation [11].

From mathematical point of view the main difficulty in solving (1) is that it is a nonlinear fourth order partial differential equation for the position vector $\mathbf{x}$ running on the surface $\mathcal{S}$. A fortunate circumstance is that this differential equation can be rewritten in the form of a system of four differential equations of second order. One, namely (1) for the mean curvature $H$ and three others, namely

$$
\Delta \mathbf{x}=2 H \mathbf{n}
$$

for the components of the position vector $\mathbf{x}$. Here $\mathbf{n}$ stands for the unit normal 
vector of $\mathcal{S}$ and the formal proof of (2) can be found either in [18] or [22].

The aim of this paper is to present explicit parametric equations describing the axisymmetric surfaces corresponding to the solutions of the shape equation (1) discovered by Naito et al. [16]. These surfaces provide the first analytical examples of surfaces with periodic curvatures studied by Kenmotsu [8]. Along this way, we have found also some unexpected relationships among Jacobian elliptic functions and their integrals.

\section{Shape Equation for Axisymmetric Vesicles}

The axisymmetric vesicles will be thought of as a surface of revolution obtained by revolving around the $z$-axis its profile curve $\Gamma$ laying in the $X O Z$-plane. If $s$ denotes the arclength along $\Gamma$ and $\psi(s)$ denotes the slope of the tangent to the curve with respect to the $O X$ axis measured counterclockwise, one has the fol-

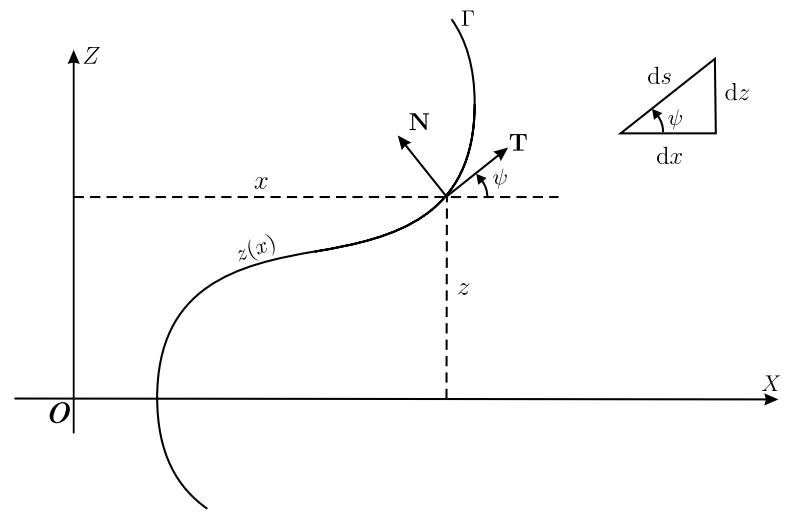

Figure 1. Geometry of the profile curve.

lowing geometrical relations

$$
\frac{\mathrm{d} \psi(s)}{\mathrm{d} s}=\kappa(s), \quad \frac{\mathrm{d} x}{\mathrm{~d} s}=\cos \psi(s), \quad \frac{\mathrm{d} z}{\mathrm{~d} s}=\sin \psi(s)
$$

which can be deduced either from Fig. 1 or the Frenet-Serret equations

$$
\frac{\mathrm{d} \mathbf{x}(s)}{\mathrm{d} s}=\mathbf{T}(s), \quad \frac{\mathrm{d} \mathbf{T}}{\mathrm{d} s}=\kappa \mathbf{N}, \quad \frac{\mathrm{d} \mathbf{N}}{\mathrm{d} s}=-\kappa \mathbf{T}
$$


in which $\mathbf{T}$ and $\mathbf{N}$ are respectively the tangent and the normal vector to the curve and $\kappa(s)$ is its curvature.

One can represent the profile curve $\Gamma$ also by the graph $(x, z(x))$ of the function $z=z(x)$ (see Fig. 1) and in the latter case the general shape equation (1) reduces to the following nonlinear third-order ordinary differential equation [5]

$$
\begin{aligned}
\cos ^{3} \psi \frac{\mathrm{d}^{3} \psi}{\mathrm{d} x^{3}}= & 4 \sin \psi \cos ^{2} \psi \frac{\mathrm{d}^{2} \psi}{\mathrm{d} x^{2}} \frac{\mathrm{d} \psi}{\mathrm{d} x}-\cos \psi\left(\sin ^{2} \psi-\frac{1}{2} \cos ^{2} \psi\right)\left(\frac{\mathrm{d} \psi}{\mathrm{d} x}\right)^{3} \\
& +\frac{7 \sin \psi \cos ^{2} \psi}{2 x}\left(\frac{\mathrm{d} \psi}{\mathrm{d} x}\right)^{2}-\frac{2 \cos ^{3} \psi}{x} \frac{\mathrm{d}^{2} \psi}{\mathrm{d} x^{2}} \\
& +\left(\frac{\lambda}{k_{c}}+\frac{\mathrm{h}^{2}}{2}-\frac{2 \mathrm{lh} \sin \psi}{x}-\frac{\sin ^{2} \psi-2 \cos ^{2} \psi}{2 x^{2}}\right) \cos \psi \frac{\mathrm{d} \psi}{\mathrm{d} x} \\
& +\left(\frac{\lambda}{k_{c}}+\frac{\mathrm{h}^{2}}{2}-\frac{\sin ^{2} \psi+2 \cos ^{2} \psi}{2 x^{2}}\right) \frac{\sin \psi}{x}+\frac{p}{k_{c}}
\end{aligned}
$$

where $\psi$ is again the angle between the $X$-axis and $\mathbf{T}$ but this time considered as a function of $x$. The two last equations in (3) imply the relation

$$
\frac{\mathrm{d} z}{\mathrm{~d} x}=\tan \psi .
$$

\section{Exact Solutions}

The general shape equation is a nonlinear fourth order partial differential equation which theory is far from being complete in any sense. As we have mentioned before, there are only a few explicit solutions which were found by relying on the axial symmetry that comprise spheres, circular cylinders [19], Clifford tori [5,20, $21]$, the rest of Delaunay constant mean curvature surfaces $[13,16]$, nodoidlike and unduloidlike shapes [16,33], and most recently the generalized cylindrical surfaces [22,27]. Even for this short list explicit parametric equations are available only for the tori [5], Delaunay [13,14] and cylindrical surfaces [27].

Many years ago, Kenmotsu [7] had shown that surfaces of a given mean curvature in $\mathbb{R}^{3}$ are defined essentially by their Gauss map (see also [4]). Later on Eells [2] pointed out that the Gauss map for Delaunay surfaces is given by the formula

$$
\sin \psi=a x+\frac{c}{x}, \quad x \neq 0, \quad a, c \in \mathbb{R} .
$$


Finally, in 1995, Naito et al. [16] discovered (see also [22]) that (7) which is solution of the shape equation (5) describing axially symmetric constant mean curvature surfaces could be generalized to the form

$$
\sin \psi=\varepsilon+\frac{1}{\ln x}+\frac{1}{4}\left(\varepsilon^{2}+2\right) \operatorname{lh} x, \quad \varepsilon \in \mathbb{R}
$$

which corresponds to vesicles with spontaneous curvature $(\mathrm{lh} \neq 0)$ subjected to nonzero pressure $(p \neq 0)$, and provided that the pressure $p$ and the tensile stress $\lambda$ are given by the expressions

$$
\frac{\lambda}{k_{c}}=\frac{\mathrm{h}^{2}\left(\varepsilon^{2}+1\right)}{2}, \quad \frac{p}{k_{c}}=-\frac{\mathrm{h}^{3}\left(\varepsilon^{2}+2\right)^{2}}{8} .
$$

For the foregoing class of solutions the equation (6) reduces to

$$
\frac{\mathrm{d} z}{\mathrm{~d} x}=\frac{\varepsilon+\frac{1}{\operatorname{lh} x}+\frac{1}{4} \operatorname{lh}\left(\varepsilon^{2}+2\right) x}{\sqrt{1-\left(\varepsilon+\frac{1}{\operatorname{lh} x}+\frac{1}{4} \operatorname{lh}\left(\varepsilon^{2}+2\right) x\right)^{2}}}
$$

and hence the profile curve of such an axisymmetric vesicle can be expressed as the graph $(x, z(x))$ of the function $z(x)$ given by the following elliptic integral

$$
z(x)=\int \frac{\varepsilon+\frac{1}{\operatorname{lh} x}+\frac{1}{4}\left(\varepsilon^{2}+2\right) \operatorname{lh} x}{\sqrt{1-\left(\varepsilon+\frac{1}{\ln x}+\frac{1}{4}\left(\varepsilon^{2}+2\right) \operatorname{lh} x\right)^{2}}} \mathrm{~d} x .
$$

The principle goal of the present paper is to find out parameterizations of the above-mentioned contours that are free of the obvious limitations associated with the graph presentations.

\section{Parametric Equations}

In terms of an appropriate new variable $u$, the equation (9) can be rewritten in the form

$$
\begin{aligned}
& \frac{\mathrm{d} x}{\mathrm{~d} u}=\frac{1}{\mu} \sqrt{-P(x) Q(x)} \\
& \frac{\mathrm{d} z}{\mathrm{~d} u}=\frac{1}{2 \mu}(P(x)+Q(x))
\end{aligned}
$$

in which

$$
\begin{aligned}
& P(x)=x^{2}+\frac{4(\varepsilon-1)}{\left(\varepsilon^{2}+2\right) \mathrm{h}} x+\frac{4}{\left(\varepsilon^{2}+2\right) \mathrm{Ih}^{2}} \\
& Q(x)=x^{2}+\frac{4(\varepsilon+1)}{\left(\varepsilon^{2}+2\right) \mathrm{h}} x+\frac{4}{\left(\varepsilon^{2}+2\right) \mathrm{Ih}^{2}}
\end{aligned}
$$


and where the real parameter $\mu$ will be fixed later on.

It should be noticed that the roots of the polynomial $\Pi(x)=P(x) Q(x)$ are

$$
\begin{array}{ll}
\alpha=\frac{2(1-\varepsilon-\sqrt{-2 \varepsilon-1})}{\left(\varepsilon^{2}+2\right) \mathrm{h}}, & \beta=\frac{2(1-\varepsilon+\sqrt{-2 \varepsilon-1})}{\left(\varepsilon^{2}+2\right) \mathrm{h}} \\
\gamma=\frac{2(-1-\varepsilon+\sqrt{2 \varepsilon-1})}{\left(\varepsilon^{2}+2\right) \mathrm{h}}, & \delta=\frac{2(-1-\varepsilon-\sqrt{2 \varepsilon-1})}{\left(\varepsilon^{2}+2\right) \mathrm{h}}
\end{array}
$$

and therefore, for each allowable value of the parameter $\varepsilon$, i.e., $|\varepsilon|>1 / 2$, only two of them are real. These are $\alpha$ and $\beta \neq \alpha$ for $\varepsilon<-1 / 2$ and, alternatively, $\gamma$ and $\delta \neq \gamma$ for $\varepsilon>1 / 2$. In the first case we will have $0<\alpha \leq x \leq \beta$ when Ih $>0$, and in the second case $x$ will be strictly positive i.e., $0<\gamma \leq x \leq \delta$ iff Ih $<0$.

Now, using the standard procedure for handling elliptic integrals (see [30], § 22.7), one can express the solution $x(u)$ of equation (10) in the form (see also [28])

$$
x(u)=\frac{2 \operatorname{sign}(\varepsilon)}{\mathrm{h} \sqrt{\varepsilon^{2}+2}}\left(1-\frac{2 \tau}{\tau+\operatorname{cn}(u, k)}\right)
$$

where

$$
\tau=\sqrt{\frac{1+|\varepsilon|+\sqrt{2+\varepsilon^{2}}}{1+|\varepsilon|-\sqrt{2+\varepsilon^{2}}}}, \quad k=\sqrt{\frac{1}{2}-\frac{3}{4 \sqrt{2+\varepsilon^{2}}}} .
$$

Actually, the choice of $u$ as uniformization variable fixes also the value of the free parameter $\mu$, i.e.,

$$
\mu=\frac{4}{\mathrm{~h}\left(2+\varepsilon^{2}\right)^{3 / 4}} .
$$

Consequently, using expressions (12) and (13), one can write down the solution $z(u)$ of equation (11) in the form

$$
z(u)=\frac{1}{\mu} \int\left(x^{2}(u)+\frac{4 \varepsilon x(u)}{\left(\varepsilon^{2}+2\right) \mathbb{h}}+\frac{4}{\left(\varepsilon^{2}+2\right) \mathbb{h}^{2}}\right) \mathrm{d} u
$$

and following this route in [28] we have found that

$$
z(u)=\mu\left[E(\operatorname{am}(u, k), k)-\frac{\operatorname{sn}(u, k) \operatorname{dn}(u, k)}{\tau+\operatorname{cn}(u, k)}-\frac{u}{2}\right] .
$$

The meaning of the functions that appear in the above equation is as follows. $E(\cdot, \cdot)$ denotes the incomplete elliptic integral of the second kind which depends 
on its argument in the first slot and the so called elliptic modulus in the second slot. The Jacobian amplitude function $\operatorname{am}(\cdot, \cdot)$ and Jacobian elliptic functions $\operatorname{sn}(\cdot, \cdot)$, $\operatorname{cn}(\cdot, \cdot)$ and $\operatorname{dn}(\cdot, \cdot)$ depend in the same manner. More details on the subject of elliptic integrals and functions can be found in [6].

In what follows we will present an alternative parameterization of Delaunay like surfaces which we hope will be of some help in their studies from the geometrical viewpoint.

We start with rewriting (14) in the form

$$
x(u)=-\frac{2 \operatorname{sign}(\varepsilon)(1+|\varepsilon|+\sqrt{2|\varepsilon|-1}) \operatorname{dn}(\tilde{u}, m)}{\left(\varepsilon^{2}+2\right) \mathrm{hh}}
$$

where

$$
\tilde{u}=\frac{K(m)}{2 K(k)} u+K(m), \quad m=\frac{2 \sqrt{(1+|\varepsilon|) \sqrt{2|\varepsilon|-1}}}{1+|\varepsilon|+\sqrt{2|\varepsilon|-1}}
$$

and $K(\cdot)$ denotes the complete elliptic integral of the first kind evaluated for the respective elliptic modulus.

Now, the remaining integrations in (15) are straightforward provided one takes into account that we have the formulas

$$
\int \mathrm{dn}(t, k) \mathrm{d} t=\operatorname{am}(t, k), \quad \int \mathrm{dn}^{2}(t, k) \mathrm{d} t=E(\operatorname{am}(t, k), k) .
$$

Actually, the integration produces the primitive

$$
\begin{aligned}
\zeta(u)= & \frac{8 K(k)}{\mu \mathrm{h}^{2}\left(\varepsilon^{2}+2\right) K(m)}\left[\frac{(1+|\varepsilon|+\sqrt{2|\varepsilon|-1})^{2}}{\varepsilon^{2}+2} E(\operatorname{am}(\tilde{u}, m), m)\right. \\
& \left.+\frac{2 \operatorname{sign}(\mathbb{h}) \varepsilon(1+|\varepsilon|+\sqrt{2|\varepsilon|-1})}{\varepsilon^{2}+2} \operatorname{am}(\tilde{u}, m)+F(\operatorname{am}(\tilde{u}, m), m)\right]
\end{aligned}
$$

in which the integration constant is omitted because if we want the sought-after curve to start from the $X$ axis for $u=0$, then obviously we have to take

$$
z(u)=\zeta(u)-\zeta(0) .
$$

Thus, for each pair of the allowed values of the parameters $\varepsilon$ and $\mathrm{Th}$, the expressions in (17) and (21) provide the parametric equations of the profile curves of 

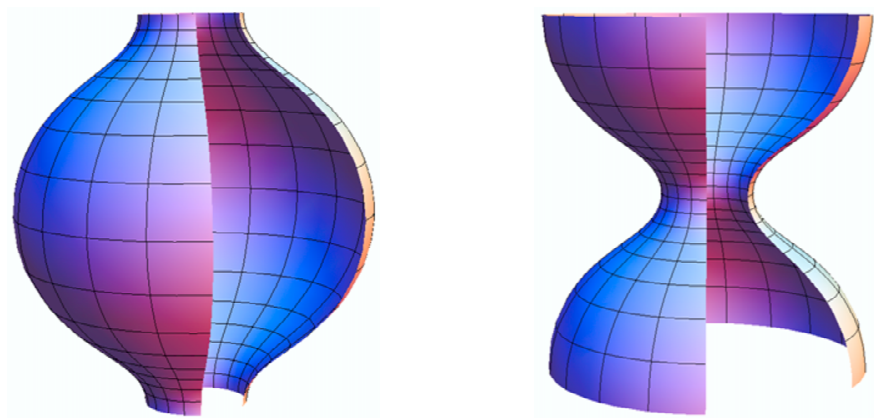

Figure 2. Open parts of the bulb (left) and the neck (right) segments of the periodic surface of revolution obtained via parametric equations (17) and (21) with $\varepsilon=1.3542$ and $\mathrm{lh}=-3.335623$.

our axially symmetric unduloid-like surfaces corresponding to the respective solutions of the membrane shape equation (5) of the form (8) (see Fig.2).

Before closing this paper, we will make the following comments. The first one is that if we equate the right hand sides of the equations (14) and (17), respectively (16) and (21) we will face quite nontrivial relationships among elliptic functions and integrals. It is hardly to believe that they could be derived in purely analytic way and probably should be considered just as glimpses of geometry.

The second one concerns the studies of the surfaces of revolution with periodic mean curvature undertaken by Kenmotsu [8] who had presented numerical examples of such surfaces. According to the authors knowledge the surfaces presented here provide the first examples from this class in analytical form.

\section{Acknowledgments}

This research is partially supported by the contract \# 35/2009 within inter-academy agreement between Bulgarian and Polish Academies of Sciences. One of the present authors (M. H.) would like to acknowledge the support from the Operational Programme "Human Resources Development" - \# BG051PO001-3.3.04/42, financed by the European Union through the European Social Fund. 


\section{References}

[1] Božič B., Svetina S., Žekš B. and Waugh R., Role of Lamellar Membrane Structure in Tether Formation From Bilayer Vesicles, Biophys. J. 61 (1992) 963-73.

[2] Eells J., The Surfaces of Delaunay, Math. Intelligencer 9 (1987) 53-57.

[3] Helfrich W., Elastic Properties of Lipid Bilayers: Theory and Possible Experiments, Z. Naturforsch 28 c (1973) 693-703.

[4] Hoffman D. and Osserman R., The Gauss Mapp of Surfaces in $\mathbb{R}^{3}$ and $\mathbb{R}^{4}$, Proc. London Math. Soc. 50 (1985) 27-56.

[5] Hu J.-G. and Ou-Yang Z.-C., Shape Equations of the Axisymmetric Vesicles Phys. Rev. E 47 (1993) 461-467.

[6] Jahnke E, Emde F. and Lösch F., Tafeln Höherer Funktionen, Teubner, Stuttgart, 1960.

[7] Kenmotsu K., Surfaces of Revolution with Prescribed Mean Curvature, Tohoku Math. J. 32 (1980) 147-153.

[8] Kenmotsu K., Surfaces of Revolution with Periodic Mean Curvature, Osaka J. Math. 40 (2003) 687-696.

[9] Konopelchenko B., On Solutions of the Shape Equation for Membranes and Strings, Phys. Lett. B 414 (1997) 58-64.

[10] Lipowsky R. and Sackmann E. (Eds), Handbook of Biological Physics (Structure and Dynamics of Membranes ) vol 1, Elsevier, Amsterdam, 1995.

[11] Liu Q.-H., Zhou H. Liu J.-X. and Ou-Yang Z.-C., Spheres and Prolate and Oblate Ellipsoids From an Analytical Solution of the SpontaneousCurvature Fluid-Membrane Model, Phys. Rev. E 60 (1999) 3227-3233.

[12] Miao L., Seifert U., Wortis M. and Dobereiner H.-G., Budding Transitions of Fluid-Bilayer Vesicles: The Effect of Area-Difference Elasticity, Phys. Rev. E 49 (1994) 5389-5407.

[13] Mladenov I., New Solutions of the Shape Equation, Eur. Phys. J. B 29 (2002) 327-330.

[14] Mladenov I., Hadzhilazova M., Djondjorov P. and Vassilev V., On the Intrinsic Equation Behind the Delaunay Surfaces, In: AIP Conference Proceedings vol. 1079, Melville-New York, 2008 pp 274-280; doi:10.1063/1.3043869.

[15] Naito H., Okuda M. and Ou-Yang Z.-C., Counterexample to Some Shape Equations for Axisymmetric Vesicles, Phys. Rev. E 48 (1993) 2304-2307. 
[16] Naito H., Okuda M. and Ou-Yang Z.-C., New Solutions to the Helfrich Variation Problem for the Shapes of Lipid Bilayer Vesicles: Beyond Delaunay's Surfaces, Phys. Rev. Lett. 74 (1995) 4345-4348.

[17] Naito H., Okuda M. and Ou-Yang Z.-C., Polygonal Shape Transformation of a Circular Biconcave Vesicle Induced by Osmotic Pressure, Phys. Rev. E 54 (1996) 2816-2826.

[18] Nitsche J., Boundary value Problems for Variational Integrals involving Surface Curvatures, Quaterly Appl. Math. 60 (1993) 363-387.

[19] Ou-Yang Z.-C. and Helfrich W., Bending Energy of Vesicle Membranes: General Expressions for the First, Second and Third Variation of the Shape Energy and Applications to Spheres and Cylinders, Phys. Rev. A 39 (1989) 5280-5288.

[20] Ou-Yang Z.-C., Anchor Ring-vesicle Membranes, Phys. Rev. A 41 (1990) 4517-4520.

[21] Ou-Yang Z.-C., Selection of Toroidal Shape of Partially Polymerized Membranes Phys. Rev. E 47 (1993) 747-749.

[22] Ou-Yang Z.-C., Liu J.-X., and Xie Y.-Z., Geometric Methods in the Elastic Theory of Membranes in Liquid Crystal Phases, World Scientific, Hong Kong, 1999.

[23] Sheetz M. and Singer S., Biological Membranes as Bilayer Couples. A Molecular Mechanism of Drug-Erythrocyte Interactions, Proc. Natl. Acad. Sci. USA 71 (1974) 4457-4461

[24] Svetina S. and Žekš B., Bilayer Couple Hypothesis of Red Cell Shape Transformations and Osmotic Hemolysis, Biomed. Biochim. Acta 42 (1983) 8690.

[25] Svetina S. and Žekš B., Membrane Bending Energy and Shape Determination of Phospholipid Vesicles and Red Blood Cells, Eur. Biophys. J. 17 (1989) 101-111.

[26] Tu Z.-C. and Ou-Yang Z.-C., Geometric Theory on the Elasticity of Biomembranes J. Phys. A: Math. \& Gen. 37 (2004) 11407-11429.

[27] Vassilev V., Djondjorov P. and Mladenov I., Cylindrical Equilibrium Shapes of Fluid Membranes, J. Phys. A: Math. \& Theor. 41 (2008) 435201 (16pp); doi:10.1088/1751-8113/41/43/435201.

[28] Vassilev V., Djondjorov P. and Mladenov I., Explicit Parametrization of Equilibrium Shapes of Axisymmetric Fluid Membranes, submitted to Phys. Rev. E. 
[29] Vassilev V. and Mladenov I., Geometric Symmetry Groups, Conservation Laws and Group-Invariant Solutions of the Willmore Equation, In: Proceedings of the Fifth International Conference on Geometry, Integrability and Quantization, SOFTEX, Sofia 2004, pp 246-265.

[30] Whittaker E. and Watson G., A Course of Modern Analysis, Cambridge University Press, Cambridge, 1922.

[31] Wiese W., Harbich W. and Helfrich W., Budding of Lipid Bilayer Vesicles and Flat Membranes, J. Phys.: Condens. Matter 4 (1992) 1647-1657.

[32] Willmore T., Riemannian Geometry, Clarendon, Oxford, 1993.

[33] Yu D. and Wang S., An Explicit Description of Axisymmetric Vesicles in Terms of Elliptic Function, Mod. Phys. Lett. B 14 (2000) 1093-1098.

Peter A. Djondjorov

Institute of Mechanics

Bulgarian Academy of Sciences

Acad. G. Bonchev Str., Bl. 4

1113 Sofia, BULGARIA

E-mail address: padjon@ imbm.bas.bg

Mariana Ts. Hadzhilazova

Institute of Biophysics

Bulgarian Academy of Sciences

Acad. G. Bonchev Str., Bl. 21

1113 Sofia, BULGARIA

E-mail address: murryh@bio21.bas.bg

Ivaïlo M. Mladenov

Institute of Biophysics

Bulgarian Academy of Sciences

Acad. G. Bonchev Str., B1. 21

1113 Sofia, BULGARIA

E-mail address: mladenov@bio21.bas.bg

Vassil M. Vassilev

Institute of Mechanics

Bulgarian Academy of Sciences

Acad. G. Bonchev Str., Bl. 4

1113 Sofia, BULGARIA

E-mail address: vasilvase imbm.bas.bg 